\title{
Three-dimensional Modelling on the Hydrodynamics of a Circulating Fluidised Bed
}

\author{
Lindsay-Marie Armstrong \\ Energy Technology Research Group \\ University of Southampton \\ Southampton, SO17 1BJ \\ United Kingdom
}

\author{
Sai Gu \\ Energy Technology Research Group \\ University of Southampton \\ Southampton, SO17 1BJ \\ United Kingdom
}

\author{
Kai H. Luo \\ Energy Technology Research Group \\ University of Southampton \\ Southampton, SO17 1BJ \\ United Kingdom
}

\begin{abstract}
The rapid depletion of oil and the environmental impact of combustion has motivated the search for clean combustion technologies. Fluidised bed combustion (FBC) technology works by suspending a fuel over a fast air inlet whilst sustaining the required temperatures. Using biomass or a mixture of coal/biomass as the fuel, FBC provides a low-carbon combustion technology whilst operating at low temperatures.

Understanding the hydrodynamic processes in fluidised beds is essential as the flow behaviours causing heat distributions and mixing determine the combustion processes. The inlet velocities and different particle sizes influence the flow behaviour significantly, particularly on the transition from bubbling to fast fluidising regimes. Computational modelling has shown great advancement in its predictive capability and reliability over recent years. Whilst 3D modelling is preferred over 2D modelling, the majority of studies use 2D models for multiphase models due to computational cost consideration.

In this paper, two-fluid modelling (TFM) is used to model a 3D circulating fluidised bed (CFB) initially focussing on fluid catalytic cracker (FCC) particles. The transition from bubbling to fast fluidisation over a range of velocities is explored, whilst the effects on the bubble diameter, particle distributions and bed expansion for different particle properties including particle sizes are compared. Drag models are also compared to study the effects of particle clustering at the meso-scale.
\end{abstract}

Keywords: CFD, Hydrodynamics, Circulating Fluidised Bed, Eulerian, Drag Models, Multiphase Flow 


\section{Nomenclature}

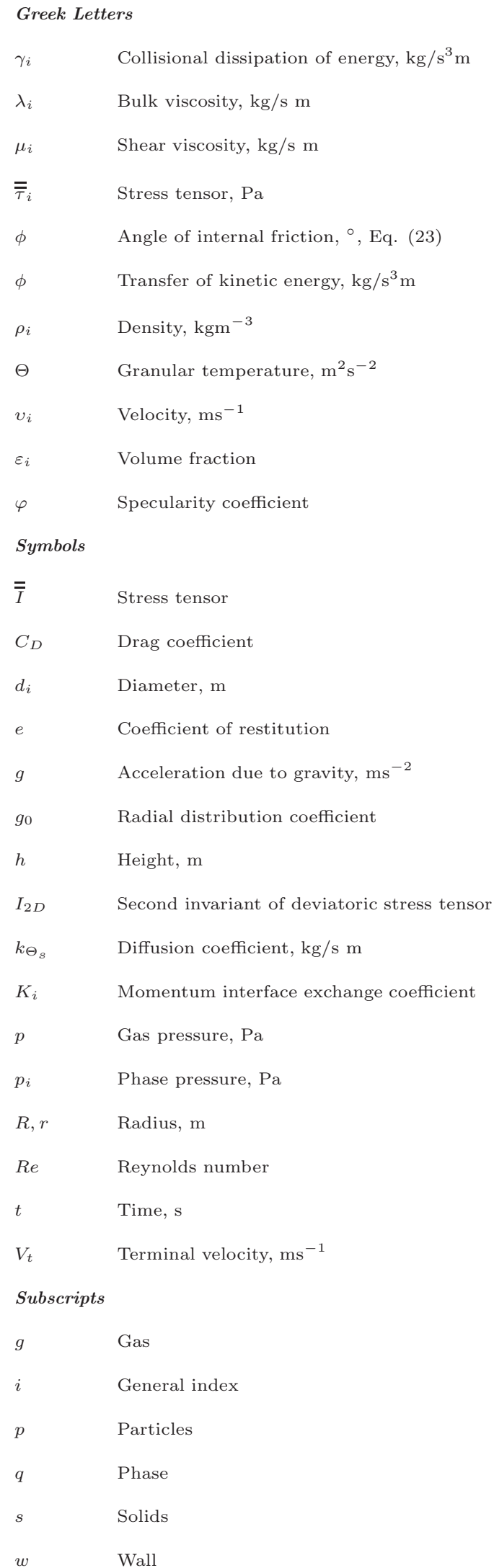

\section{Introduction}

The increasing application of circulating fluidised bed (CFB) technology in industry means optimum reactor designs are required to improve efficiency and reduce emissions. Examples include cracking, combustion and power generation $[1,2]$. Computational fluid dynamic (CFD) modelling of the dynamic behaviours has become a viable tool for simulating the processes that take place in CFBs. The increase in computer performance and capabilites allows for complex geometries, difficult experimental measuring conditions and non-invasive simulations to be carried out. Before the accurate simulations of the full reaction processes, can be carried out, the basic hydrodynamics of the flow needs to be thoroughly understood. The hydrodynamics of fluidised bed reactors has attracted a number of researchers for decades to understand the complex interactions between gas and particles $[1,2,5,17,18]$.

Most studies apply the Eulerian-Eulerian twofluid model (TFM) which assumes the gas-solid phases as continuous and fully interpenetrating within each control volume $[1,6,17,18]$. It is less computationally exhaustive in comparison to the other models: the discrete Eulerian-Lagrangian method which simulates the individual particle dynamics, and the complete Lagrangian model which models both particles and fluid with a Lagrangian approach $[3,4,5]$. However, TFMs have issues with regard to scaling sizes. In order to obtain sufficient information about particle-fluid interactions and structures, small control volumes are required. This method has shown reasonable results for small scale models with a height around $1-2 \mathrm{~m}$ as the control volumes are small. However, increasing the dimensions of the reactor would require large cell sizes to reduce computational time and expense resulting in the model's inability to capture the full particle structures [6].

The kinetic theory of granular flow is one of the most important tools for modelling the motion of particles. The basic concept of the kinetic theory of granular flow is the granular temperature. During random oscillations of the particles, inelastic collisions occur causing energy to be dissipated. The granular temperature measures these random oscillations of the particles and is defined as the average of the three variances of the particle's velocities. A full mathematical description of the kinetic theory is provided by Gidaspow [7].

The drag models are important in simulating the interphase momentum transfer between the gas and 
particle phases. There are a number of averagebased drag models available and displayed throughout literature including the Gidaspow [8] and the Syamlal [9] drag model. Whilst these models have produced similar results compared to experimental data, they do not take into account the structure of particle clusters at different scales. O'Brien et al [10] stated the importance of the clustering of particles needs to be accounted for in current drag correlations. Gunn et al [11] determined that for grouped clusters of particles with a given fluid flow rate and voidage, there was a decrease in the measured drag coefficient due to the increase of gas flowing around the clusters and decrease gas flow penetrating them. Yang et al [12] modified a drag model to incorporate the energy minimisation multiscale (EMMS) approach [13]. This approach looks at the phase interactions that take place at three different scales. Micro-scale modelling of the discrete particles in either the dilute phase which is fluid-dominated (FD) and the dense phase which is particle-dominated (PD). Meso-scale modelling of the clusters of particles with the interactions between the dense cluster and the dilute broth phase, known as particle-fluid compromising (PFC). Macro-scale modelling considers the boundary effects upon the particle-fluid suspensions and interactions. Li et al [14] found the flow to be heterogenous in gas-fluid fluidisation and the effects of the boundaries can lead to axial and radial heterogeneity. These heterogeneous structures cause issues when using standard averagedbased TFM approaches as disparity between the dilute core annulus and dense wall regions are so dramatic. The EMMS drag model has been applied by key researchers within the field of fluidisation hydrodynamics $[14,12,15]$, calculations were made and compared for the slip velocities and drag coefficients for the different interaction phases of dense clusters, dilute broth phases and interactions between them both [13]. This was further extended to show the strong dependence of the drag coefficient on simple structural differences [16] and later a decrease in drag coefficient due to local and global structural changes. The standard TFMs only relate the drag coefficients to the local slip velocities and average voidages hence not displaying the overall structural effects.

The Reynolds numbers for the multiphase flows within CFB risers are high so the application of additional turbulence models to the kinetic theory has been applied for many years $[1,17,18]$. The turbulent interaction between the phases and time averaged turbulent flow behaviour needs to be modelled with the correct closure models and empirical constants in order to accurately simulate more realistic results. However, comparisons between turbulence models and laminar models [18] suggested that the laminar models showed more consistant results over the turbulence models. These comparative models for turbulence were carried out in $2 \mathrm{D}$ simulations and since turbulence fluctuations always have threedimensional spatial character then 3D comparative models are required to draw a firm conclusion.

Three-dimensional modelling has been carried out by several researchers $[19,20,21,22]$ with results confirming $3 \mathrm{D}$ models to be superior over the 2D models. However, 2D modelling still takes preference over 3D modelling due to the excessive computational time and expense 3D modelling incurs. With the increase in computational performance and introduction of parallel computing systems, the issues with computational time for 3D modelling are reduced.

The present work initially compares an isothermal 2D CFD model using the commercial software FLUENT 6.2.16 to experimental data taken for a CFB model from literature [1]. The regimes of fluidisation are explored for a range of inlet velocities and particle diameters. The distribution and size of air bubbles throughout the bed are examined for bubbling fluidisation through to the fast fluidisation. Radial particle velocities are compared to experimental results with volume fraction down a CFB wall explored. Finally, 3D modelling is carried out to show the bubble formations and particle clustering within both regimes. The distribution of bubble sizes is investigated for a range of velocities and results are compared to those obtain from the 2D modelling.

\section{Experimental Setup}

The laboratory reactor used for the experiment is provided in more detail by Samuelsberg and Hjertager[1]. Figure 1 shows a sketch of the experimental set up. The root mean square velocities of the particles were taken at three heights, $0.16 \mathrm{~m}$, $0.32 \mathrm{~m}$ and $0.48 \mathrm{~m}$ in the reactor using LDA technology. Once fluidised, the particles would travel up the reactor and into the cyclone. Gas would exit from the top of the cyclone whilst particles descend down the downcomer to be re-introduced into the reactor. An initial static bed height for the catalyst particles was $0.05 \mathrm{~m}$ high with a secondary air inlet position at the same height. The secondary inlet forced circulating particles back into the reactor. 
FCC particles were used with a density of $1600 \mathrm{~kg} / \mathrm{m}^{3}$ and a diameter range $20 \mu \mathrm{m}-150 \mu \mathrm{m}$. The mean diameter was taken to be $60 \mu \mathrm{m}$. Two superficial gas velocities were introduced through the primary inlet, $0.71 \mathrm{~ms}^{-1}$ and $1.42 \mathrm{~ms}^{-1}$ at an ambient temperature. The secondary inlet introduced air at a constant rate of $0.05 \mathrm{~ms}^{-1}$.

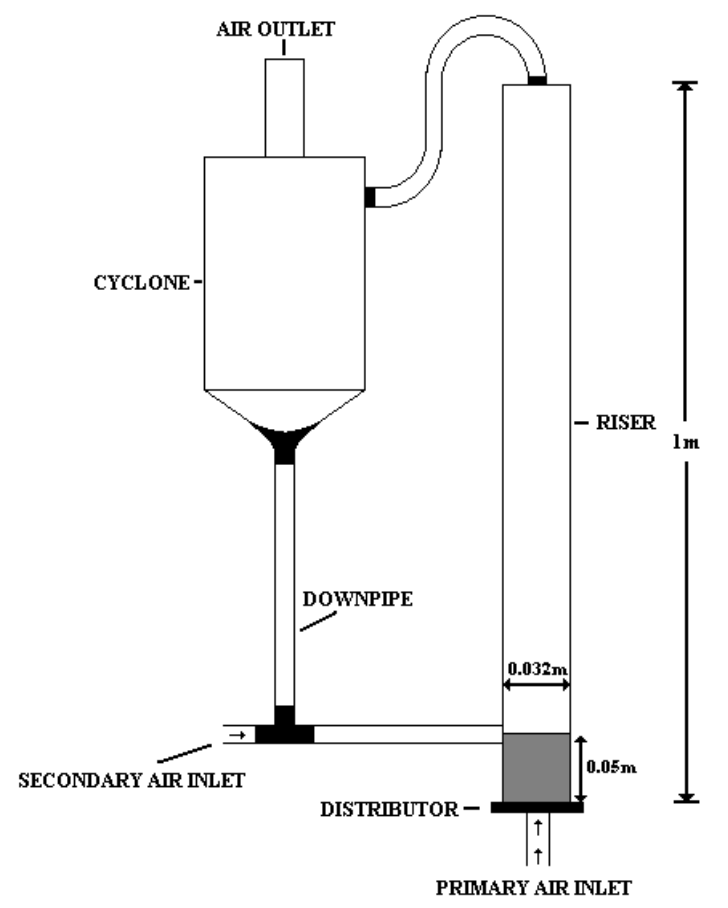

Figure 1: Sketch of the laboratory scale circulating fluidised bed used in literature[1]

\section{CFD Modelling}

\subsection{Mesh production}

A 2D mesh was produced in Gambit containing 78934 cells of width $0.001 \mathrm{~m}$ with the reactor made up from $28 \times 669$ nodes in the radial and axial directions, respectively. To capture the complex flow behaviours at the walls, the nodes in the radial direction were non-uniformly distributed with a more refined grid near the walls. The grid in the axial direction was uniformly distributed apart from the region where the downcomer re-enters the reactor which is refined to capture the re-entry effects of the particles.

The 3D mesh contained 1620798 cells with a cell width of $0.002 \mathrm{~m}$. The walls were refined to a $0.0005 \mathrm{~m}$ cell width to accurately model the down- flow at the walls. To reduce the computational cost, the $3 \mathrm{D}$ grid is coarser than the $2 \mathrm{D}$ grid.

The bubbling bed uses the same diameter as the CFB riser but only halve the height of $0.5 \mathrm{~m}$. The 2D mesh contains 18732 cells with $38 \times 971$ nodes in the radial and axial directions. As with the CFB model, the mesh is refined at the walls. The secondary inlet is not present in the bubbling bed as the transition from bubbling to fast fluidisation is the main objective therefore the mesh contains the primary inlet, riser and a pressure outlet set $0.5 \mathrm{~m}$ above the inlet. The 3D mesh contains 733821 cells of width $0.002 \mathrm{~m}$ with the refined mesh at the walls.

\subsection{Governing equations}

The Eulerian-granular model in FLUENT 6.2.16 is used to model the interactions between gas and granular particles within this fluidised bed. This model allows for the presence of two different phases in one control volume of the grid by introducing the volume fraction variable. The solid phase contains spherical granular particles of the same diameter. These two phases are solved individually using the mass and momentum equations. Table 1 gives details of the full equations. The kinetic fluctuations between particles is considered using the kinetic theory of granular flow given in Table 1 . The energy equation is ignored in this case as the flow is isothermal as are the virtual mass and lift effects. This is because lift only effects particles of large diameters and this is not the present case.

The gas-solid interphase exchange coefficient, $K_{g s}$, was modelled for the bubbling fluidised beds and the initial circulating fluidised bed using the Gidaspow drag function as in Table 2. This drag function is applicable to both dense and dilute systems as it is comprised of the Wen and Yu model[23] for dilute phases and the Ergun model[24] for dense phases. The circulating fluidised bed is modelled further to account for particle clustering. This is done by coupling the EMMS model (Table 2) into Fluent with User-Defined Functions (UDF).

The solid shear viscosity is composed of collisional, kinetic and frictional effects. For very dense flows, frictional viscosity is applied due to the volume fraction for the particles approaching closely to the packing limit. The friction created between the particles generates a large amount of stress. Schaeffer's expression[27] is used to model the frictional viscosity in dense cases. The bulk viscosity accounts for the resistance of particle to expansion and depression and is calculated using an expression from Lun et al.[28]. The solids pressure is composed of 
two terms, where the first term represents the kinetic term and the second term is due to particle collisions (Equation 29). It was determined from an equation of state which was similar to the van der Waals equation of state for gases[26]. The radial distribution function (Equation 26) modifies the probability of particle collisions as the phase becomes dense. For large Reynolds numbers, the modified $\mathrm{k}-\epsilon$ model uses the standard single phase $\mathrm{k}-\epsilon$ model for the gas phase but introduces a turbulent momentum transfer term between the gas and solid phase.

The finite volume method was used to solve the governing equations. The coupling and correction of the velocity and pressure is carried out for multiphase flows with the Phase Coupled SIMPLE (PCSIMPLE) algorithm [25]. The discretisation of the convective terms was carried out with the secondorder upwind scheme. A time step of $1 \times 10^{-4}$ was used to ensure quick convergence with a maximum of 30 iterations per time step. The convergence criterion between two iterations was set to $1 \times 10^{-3}$.

\subsection{Boundary and initial conditions}

The particle bed is initially set to a height of $0.05 \mathrm{~m}$ above the main inlet with particles set to a diameter of $60 \mu \mathrm{m}$ and density of $1600 \mathrm{~kg} / \mathrm{m}^{3}$. There are two gas inlets, the main inlet providing constant gas supply with velocities $0.71 \mathrm{~ms}^{-1}$ and $1.42 \mathrm{~ms}^{-1}$, and a secondary inlet set to $0.05 \mathrm{~ms}^{-1}$ to prevent backflow of particles within the re-entering tube. A pressure outlet was used with no solid particles allowed to leave the reactor.

The effects of particle-wall collisions within CFB risers plays a significant part on the shear stress at the walls. The boundary conditions at the walls for the gas phase have tangential and normal velocities of zero, namely no-slip boundary conditions. For the particle phase, a tangential slip condition is imposed developed by Johnson and Jackson [29]. Johnson and Jackson used the coefficient of restitution for particle-wall collisions along with the specularity coefficient, which quantifies the nature of the particle-wall collisons based on whether the walls are smooth and frictionless or very rough. The granular temperature is modelled by equation (28) which is found by equating the granular temperature flux to the wall and the generation of granular temperature at the wall to the energy dissipation due to particle-wall collisions. Table 3 shows the equations used to model the slip of particles and granular temperature against the wall. The param-
Table 1: Governing Equations

$$
\begin{aligned}
& \text { Conservation of Mass } \\
& \text { Gas } \\
& \qquad \frac{\partial\left(\varepsilon_{g} \rho_{g}\right)}{\partial t}+\nabla \cdot\left(\varepsilon_{g} \rho_{g} \vec{v}_{g}\right)=0 \\
& \text { Particle } \\
& \quad \frac{\partial\left(\varepsilon_{s} \rho_{s}\right)}{\partial t}+\nabla \cdot\left(\varepsilon_{s} \rho_{s} \vec{v}_{s}\right)=0
\end{aligned}
$$$$
\text { Eqn }
$$

Conservation of Momentum

$$
\begin{aligned}
& \text { Gas } \\
& \frac{\partial\left(\varepsilon_{g} \rho_{g} \vec{v}_{g}\right)}{\partial t}+\nabla \cdot\left(\varepsilon_{g} \rho_{g}\left(\vec{v}_{g} \otimes \vec{v}_{g}\right)\right)=-\varepsilon_{g} \nabla p \\
& +\nabla \cdot \overline{\bar{\tau}}_{g}+\varepsilon_{g} \rho_{g} \vec{g}+K_{g s}\left(\vec{v}_{g}-\vec{v}_{s}\right) \\
& \frac{\partial\left(\varepsilon_{s} \rho_{s} \vec{v}_{s}\right)}{\partial t}+\nabla \cdot\left(\varepsilon_{s} \rho_{s}\left(\vec{v}_{s} \otimes \vec{v}_{s}\right)\right)=-\varepsilon_{s} \nabla p \\
& -\nabla p_{s}+\nabla \cdot \overline{\bar{\tau}}_{s}+\varepsilon_{s} \rho_{g} \vec{g}+K_{g s}\left(\vec{v}_{g}-\vec{v}_{s}\right)
\end{aligned}
$$

$$
\begin{aligned}
& \text { Phase Stress-Strain Tensor } \\
& \qquad \begin{array}{r}
\overline{\bar{\tau}}_{q}=\varepsilon_{q} \mu_{q}\left(\nabla \vec{v}_{q}+\nabla \vec{v}_{q}^{T}\right) \\
\quad+\varepsilon_{q}\left(\lambda_{q}-\frac{2}{3} \mu_{q}\right) \nabla \cdot \vec{v}_{q} \overline{\bar{I}}_{q}
\end{array}
\end{aligned}
$$

$$
\begin{aligned}
& \text { Kinetic Fluctuation Energy } \\
& \begin{array}{c}
\frac{3}{2}\left[\frac{\partial}{\partial t}\left(\varepsilon_{s} \rho_{s} \Theta_{s}\right)+\nabla \cdot\left(\varepsilon_{s} \rho_{s} \vec{v}_{s} \Theta_{s}\right)\right]=-\gamma_{\Theta s} \\
+\phi_{g s}+\left(-p_{s} \cdot \overline{\bar{I}}+\overline{\bar{\tau}}_{s}\right): \nabla \vec{v}_{s} \\
+\nabla \cdot\left(k_{\Theta_{s}} \cdot \nabla \Theta_{s}\right)
\end{array} \\
& k_{\Theta_{s}}=\frac{150 \rho_{s} d_{s} \sqrt{\Theta_{s} \pi}}{384(1+e) g_{0}}\left[1+\frac{6}{5} \epsilon_{s} g_{0}(1+e)\right]^{2} \\
& +2 \epsilon_{s}^{2} \rho_{s} d_{s}(1+e) g_{0} \sqrt{\frac{\Theta}{\pi}} \\
& \gamma_{\Theta s}=\frac{12(1-e)^{2} g_{0}}{d_{s} \sqrt{\pi}} \epsilon_{s}^{2} \rho_{s} \Theta_{s}^{3 / 2} \\
& \phi_{g s}=-3 K_{g s} \Theta_{s}
\end{aligned}
$$

Definitions

$\varepsilon_{g}+\varepsilon_{s}=1$ 
Table 2: Constitutive equations

Constitutive equations
Gidaspow Drag Model
for $\varepsilon_{g} \leq 0.8$
\[ K_{g s}=150 \frac{\varepsilon_{s}^{2} \mu_{g}}{\varepsilon_{g} d_{s}^{2}}+1.75 \frac{\varepsilon_{s} \rho_{g}\left|\overrightarrow{v_{s}}-\vec{v} g\right|}{d_{s}} \]
for $\varepsilon_{g}>0.8$
\[ K_{g s}=\frac{3}{4} C_{D} \frac{\varepsilon_{s} \varepsilon_{g} \rho_{g}\left|\overrightarrow{v_{s}}-\overrightarrow{v_{g}}\right|}{d_{s}} \varepsilon_{g}^{-2.65} \]
$C_{D}=\frac{24}{\varepsilon_{g} R e_{s}}\left[1+0.15\left(\varepsilon_{g} R e_{s}\right)^{0.687}\right]$

\section{EMMS Drag Model}

$$
\begin{aligned}
& K_{g s}=150 \frac{\varepsilon_{s}^{2} \mu_{g}}{\varepsilon_{g}^{2} d_{s}^{2}}+1.75 \frac{\varepsilon_{s} \rho_{g}\left|\overrightarrow{v_{s}}-\overrightarrow{v_{g}}\right|}{\varepsilon_{g} d_{s}} \quad \text { for } \varepsilon_{g} \leq 0.8 \\
& K_{g s}=\frac{3}{4} C_{D} \frac{\varepsilon_{s} \rho_{g}\left|\overrightarrow{v_{s}}-\overrightarrow{v_{g}}\right|}{d_{S}} \omega(\varepsilon) \quad \text { for } \varepsilon_{g}>0.8 \\
& \text { where } \\
& \omega(\varepsilon)=\left\{\begin{array}{cc}
-0.5760+\frac{0.0214}{4\left(\varepsilon_{g}-0.7463\right)^{2}+0.0044} & \left(0.8<\varepsilon_{g} \leq 0.82\right) \\
-0.0101+\frac{0.0038}{4\left(\varepsilon_{g}-0.7789\right)^{2}+0.0040} & \left(0.82<\varepsilon_{g} \leq 0.97\right) \\
-31.8295+32.8295 \varepsilon_{g} & \left(\varepsilon_{g}>0.97\right)
\end{array}\right. \\
& C_{D}=\frac{24}{\operatorname{Re}_{S}}\left[1+0.15\left(R e_{S}\right)^{0.687}\right] \text { for } R e_{S} \leq 1000 \\
& C_{D}=0.44 \text { for } \operatorname{Re}_{S}>1000 \\
& \operatorname{Re}_{s}=\frac{\rho_{g} d_{s}\left|\overrightarrow{v_{s}}-\overrightarrow{v_{g}}\right|}{\mu_{g}}
\end{aligned}
$$

Solids Shear Viscosity

$$
\mu_{s}=\mu_{s, \mathrm{col}}+\mu_{s, \mathrm{kin}}+\mu_{s, \mathrm{fr}}
$$

\section{Collisional Viscosity}

$$
\mu_{s, \mathrm{col}}=\frac{4}{5} \varepsilon_{s} d_{s} \rho_{s} g_{0}(1+e)\left(\frac{\Theta_{s}}{\pi}\right)^{1 / 2}
$$

\section{Kinetic Viscosity}

$$
\mu_{s, \mathrm{kin}}=\frac{10 d_{s} \rho_{s} \sqrt{\Theta_{s} \pi}}{96 \varepsilon_{s} g_{0}(1+e)}\left[1+\frac{4}{5} \varepsilon_{s} g_{0}(1+e)\right]^{2}
$$

\section{Frictional Viscosity}

$$
\mu_{s, \mathrm{fr}}=\frac{p_{s} \sin \phi}{2 \sqrt{I_{2 D}}}
$$

\section{Solid Bulk Viscosity}

$$
\lambda_{s}=\frac{4}{3} \varepsilon_{s} d_{s} \rho_{s} g_{0}(1+e)\left(\frac{\Theta_{s}}{\pi}\right)^{1 / 2}
$$

\section{Particle Pressure}

$$
p_{s}=\varepsilon_{s} \rho_{s} \Theta_{s}+2 \rho_{s}(1+e) \varepsilon_{s}^{2} g_{0} \Theta_{s}
$$

\section{Radial Distribution Function}

$$
g_{0}=\left[1-\left(\frac{\varepsilon_{s}}{\varepsilon_{S}, \max }\right)^{1 / 3}\right]^{-1}
$$

Table 3: Boundary Conditions

Particle Phase Boundary Conditions

\section{Velocity

$$
\vec{u}_{s, w}=-\frac{6 \mu_{s} \varepsilon_{s, \max }}{\sqrt{3} \sqrt{\theta} \pi \varphi \rho_{s} \varepsilon_{s} g_{0}} \frac{\partial \vec{v}_{s, w}}{\partial n}
$$

Granular Temperature

$$
\begin{aligned}
& \theta_{w}=-\frac{\kappa \theta}{\gamma w} \frac{\partial \theta}{\partial n}+\frac{\sqrt{3} \pi \varphi \rho_{s} \varepsilon_{s} g_{0} \vec{v}_{s, \text { slip }}^{2} \theta^{\frac{3}{2}}}{6 \gamma \boldsymbol{w}_{s}, \text { max }} \\
& \gamma_{w}=\frac{\sqrt{3} \pi\left(1-e_{w}^{2}\right) \varepsilon_{s} \rho_{s} g_{0} \theta^{\frac{3}{2}}}{4 \varepsilon_{s, \text { max }}}
\end{aligned}
$$

Table 4: Table of Parameters

\section{Gas}

\begin{tabular}{llr}
$v_{g}$ & Velocity & $(0.06-1.42) \mathrm{ms}^{-1}$ \\
$\rho_{g}$ & Density & $1.225 \mathrm{kgm}^{-3}$ \\
$\mu_{g}$ & Shear viscosity & $1.79 \times 10^{-5} \mathrm{~kg} / \mathrm{m} \mathrm{s}$ \\
& \\
\hline & \\
\hline
\end{tabular}

$\begin{array}{llr}d_{p} & \text { Particle diameter } & 40,60,80 \mu \mathrm{m} \\ \rho_{p} & \text { Particle density } & 1600 \mathrm{kgm}^{-3} \\ e & \text { Particle coef. of restitution } & 0.995 \\ e_{w} & \text { Wall coef. of restitution } & 0.95 \\ \varphi & \text { Specularity coefficient } & 0.25\end{array}$


eters used within the simulations are provided in Table 4 .

\section{Results and Discussion}

\subsection{Bubbling fluidised beds}

\subsubsection{D Bubbling fluidised beds}

Initially, mono-sized particles with diameter $60 \mu \mathrm{m}$ were modelled within the riser section of a CFB from literature [1]. The secondary air inlet was neglected as the transition from bubbling to fast fluidisation is the focus of the initial study. The simulations concentrated on the lower $0.5 \mathrm{~m}$ region of the riser and ran for $9 \mathrm{~s}$ to allow for complete fluidisation. The volume fraction distribution for the particles with four inlet velocities are shown in Fig. 2(a-d). For particles with a diameter $60 \mu \mathrm{m}$, the terminal velocity, $\mathrm{V}_{t}$, is $0.175 \mathrm{~ms}^{-1}$ so inlet velocities were chosen to capture the behaviour of the particles before and after $\mathrm{V}_{t}$. Although the terminal velocity applies to single particles in suspension, the bursting-bubbles at the top of the particle bed blow a small number of particles above the bed where they are effectively suspended. If the gas velocity does not exceed $\mathrm{V}_{t}$ the particles fall back down to the particle bed, referred to as bubbling beds and displayed in Fig. 2(a)(b). Exceeding $\mathrm{V}_{t}$ means the suspended particles can be carried with the gas phase and continue up the riser. This fast fluidisation state can be seen in Fig. 2(c)(d).

The bed height expansions for the bubbling fluidised states are $0.14 \mathrm{~m}$ and $0.298 \mathrm{~m}$ for $0.1 \mathrm{~ms}^{-1}$ and $0.16 \mathrm{~ms}^{-1}$, respectively. The bubbles appear smaller and regularly shaped near the entrance of the riser, increasing in size and distorting with increasing height. As the velocity increases, the bubble size increase and the solid-gas mixture appears more dilute. The fast fluidising state shows very dilute results however the lower velocity shows a higher accumulation of particles nearer the inlet.

The variation of particle diameter results in the change in $\mathrm{V}_{t}$. A further two diameters were simulated, $40 \mu \mathrm{m}$ and $80 \mu \mathrm{m}$. Figures $2(\mathrm{e})(\mathrm{f})$ and $2(\mathrm{~g})(\mathrm{h})$ show the results above and below their terminal velocities of $0.0778 \mathrm{~ms}^{-1}$ and $0.3113 \mathrm{~ms}^{-1}$, respectively. Reduction in particle diameter shows smaller bubbles whereas the bubble size appears to be increased with larger diameters. The results agree with the theory that a velocity higher than $\mathrm{V}_{t}$ results in a fast fluidising regime whereas a

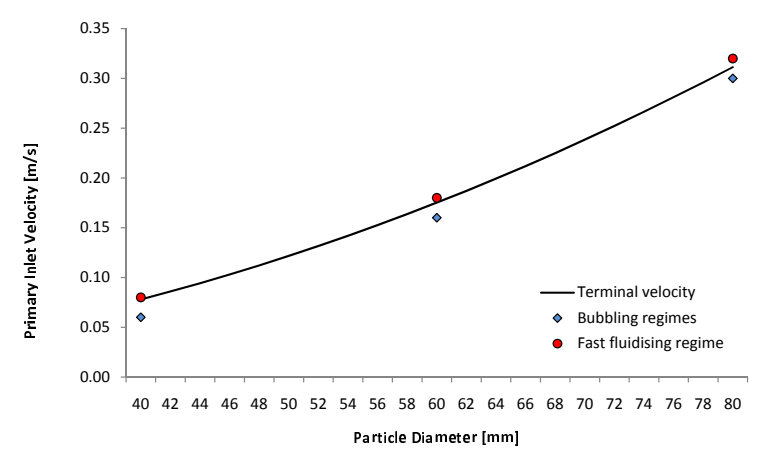

Figure 3: Fast fluidising regimes observed above the terminal velocity, $\mathrm{V}_{t}$, and bubbling fluidised regimes below the $\mathrm{V}_{t}$

velocity lower has a bubbling fluidising regime. Figure 3 displays the graph of the terminal velocity for a range of diameters. The inlet velocities for the 3 diameter particles are plotted and coloured to show the fast fluidising regimes occuring above $\mathrm{V}_{t}$ and bubbling regimes lying below $\mathrm{V}_{t}$.

\subsubsection{D Bubbling fluidised beds}

Three dimensional models were performed on two cases; case $1: \mathrm{d}_{p}=60 \mu \mathrm{m}$ with $\mathrm{V}=0.16 \mathrm{~ms}^{-1}$ and case $2: \mathrm{d}_{p}=80 \mu \mathrm{m}$ with $\mathrm{V}=0.3 \mathrm{~ms}^{-1}$. The volume fraction of particles across different slices of the bubbling beds are displayed in Fig. 4 and Fig. 5 for case 1 and 2 respectively.

The model with particle diameter $60 \mu \mathrm{m}$ shows

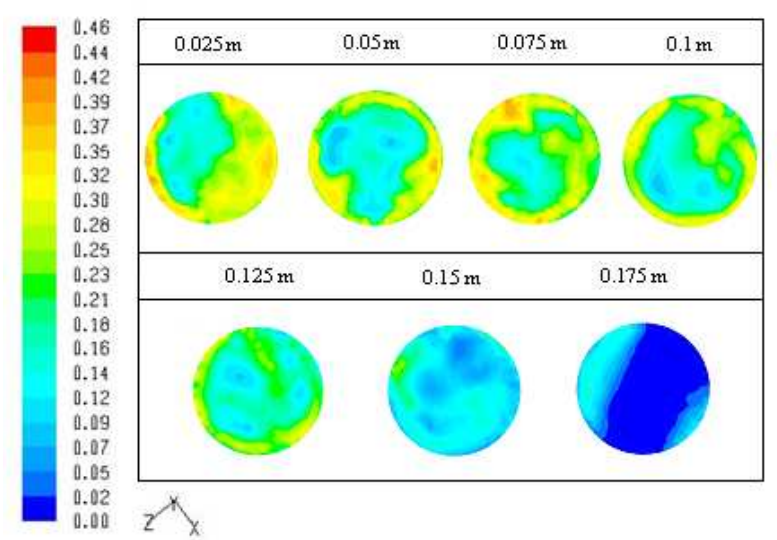

Figure 4: Volume fraction of $60 \mu \mathrm{m}$ particles at different heights of the riser

the expected particle segregation towards the walls 


\begin{tabular}{|c|c|c|c|c|c|c|c|c|}
\hline \multirow{2}{*}{$\begin{array}{l}\text { VOLUME } \\
\text { FRACTION }\end{array}$} & \multicolumn{4}{|c|}{ DIAMETER $=60 \mu \mathrm{m}$} & \multicolumn{2}{|c|}{ DIAMETER $=40 \mu \mathrm{m}$} & \multicolumn{2}{|c|}{ DIAMETER $=80 \mu \mathrm{m}$} \\
\hline & a) & b) & c) & d) & e) & f) & g) & h) \\
\hline 0.50 & & & & & & & & \\
\hline 0.47 & & & & & & & & \\
\hline 0.43 & & & & & & & & \\
\hline 0.40 & & & & & & & & \\
\hline 0.38 & & & & & & & & \\
\hline 0.32 & & & & & & & & \\
\hline 0.30 & & & & & & & & \\
\hline 0.28 & & & & & & & & \\
\hline $\begin{array}{l}0.25 \\
0.22\end{array}$ & & & & & & & & \\
\hline 0.20 & & & & & & & & \\
\hline 0.17 & & a & & & & & & \\
\hline 0.15 & 8 & & & & & & & \\
\hline 0.13 & 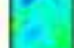 & 87 & & & & & & \\
\hline 0.08 & & & & & & & & \\
\hline 0.05 & 8 & & & & & & $r$ & a \\
\hline 0.03 & & ? & & & 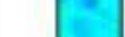 & & & \\
\hline 0.00 & 1. 52 & 12) & 91 & & L. I I & & & IIA \\
\hline
\end{tabular}

Figure 2: Volume fraction of particles at 9.0s with 3 different diameters over a range of velocities above and below their terminal velocities. For $\mathrm{d}_{p}=60 \mu \mathrm{m}$ : a) $\mathrm{V}=0.1 \mathrm{~ms}^{-1}$, b) $\mathrm{V}=0.16 \mathrm{~ms}^{-1}$, c) $\mathrm{V}=0.18 \mathrm{~ms}^{-1}$ and d) $\mathrm{V}=0.36 \mathrm{~ms}^{-1}$. For $\mathrm{d}_{p}=$ $40 \mu \mathrm{m}:$ e) $\mathrm{V}=0.06 \mathrm{~ms}^{-1}$ and f) $\mathrm{V}=0.08 \mathrm{~ms}^{-1}$. For $\left.\mathrm{d}_{p}=80 \mu \mathrm{m}: \mathrm{g}\right) \mathrm{V}=0.3 \mathrm{~ms}^{-1}$ and $\left.\mathrm{h}\right) \mathrm{V}=0.32 \mathrm{~ms}^{-1}$

with the dilute region situated within the central core of the bed. The contours are taken to a height of $0.175 \mathrm{~m}$ as the region above is highly diluted. The denser region of particles are observed at the base of the bed becoming more dilute with increasing height.

Case 2 showed generally a more dilute distribution of particle throughout the bed compared to the distribution of the $60 \mu \mathrm{m}$ particles, indicating that the voidages are larger throughout the bed as the diameter of particles increases. This is observed also in the two dimensional results also (fig 2 ). The dilute core is present with the accumulation of particles around the walls. At lower heights, the bed is denser. The accummulated particles on the walls descend back down to the lower bed continually sustaining this denser region. As the bubbles rise, they become enlarged as the coalescence of the faster smaller bubbles from the lower heights catch up to the larger voidages near the top of the bed. This is observed in both cases as the mixture becomes more dilute with ascending height indicating larger voidages are present.

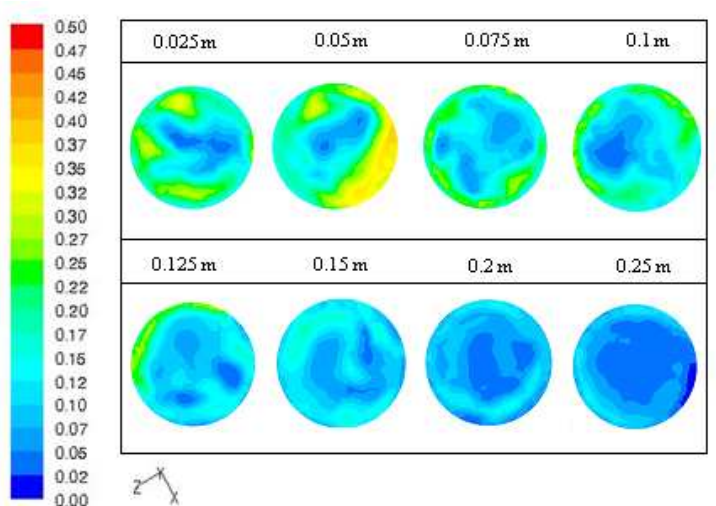

Figure 5: Volume fraction of $80 \mu \mathrm{m}$ particles at different heights of the riser 


\subsection{Circulating Fluidised beds}

\subsubsection{D circulating fluidised bed}

The complete CFB was modelled with much faster velocities than those tested for the bubbling beds, at $0.71 \mathrm{~ms}^{-1}$ and $1.42 \mathrm{~ms}^{-1}$. The $2 \mathrm{D}$ radial particle velocities were taken at three heights in the riser after $14.0 \mathrm{~s}$ for velocity $1.42 \mathrm{~ms}^{-1}$ as shown in fig. 6. The results compare well to the experimental results extracted from literature[1]. However, the experimental data appeared to be taken from half the diameter and replicated symetrically to give the symmetrical results shown. The simulated results show a slightly skewed velocity within the core due to the position of the secondary air inlet. The recirculated air enters from a side angle causing the flow to become slightly asymmetric. The transitional flow structure would also affect the particle distribution within the gas flow which can be seen in Fig. 6 and literature [1].

The velocity of the particles within the central core is slightly over predicted, however the wall effects show reasonable predictions which are better than those in the literature [1]. This is because the specularity coefficient was set to $\varphi=0.25$ and not allowing a complete free slip wall $(\varphi=0)$. The particles at the walls descend back down the pipe due to the dense particle flow. The narrowing of the core diameter can be seen at all three heights and this explains the overestimation of the core velocity. The core diameter at $0.16 \mathrm{~m}$ is narrower than the other heights as the build up of particles is larger against the wall. This is shown in Fig. 7 which displays the volume fraction of particles at the walls across the 3 heights. The volume fraction at the walls is higher at the lower height as particles continually descend down the walls whilst accummulating more particles from the core. There is a slight increase in volume fraction within the core particularly with increasing height as the dispersed particles are carried with the flow. The values are however significantly lower then the regions against the wall.

\subsubsection{D circulating fluidised bed}

The 3D models were run for 15.0s using both the Gidaspow drag model and the EMMS model. Figure 8 compares the two drag models within the lower $0.4 \mathrm{~m}$ of the riser at $15.0 \mathrm{~s}$. The contour plot of the volume fraction shows the EMMS model correctly models the dense collection of particles at the base of the riser. Furthermore, clusters of particles on a mesoscale are taken into account
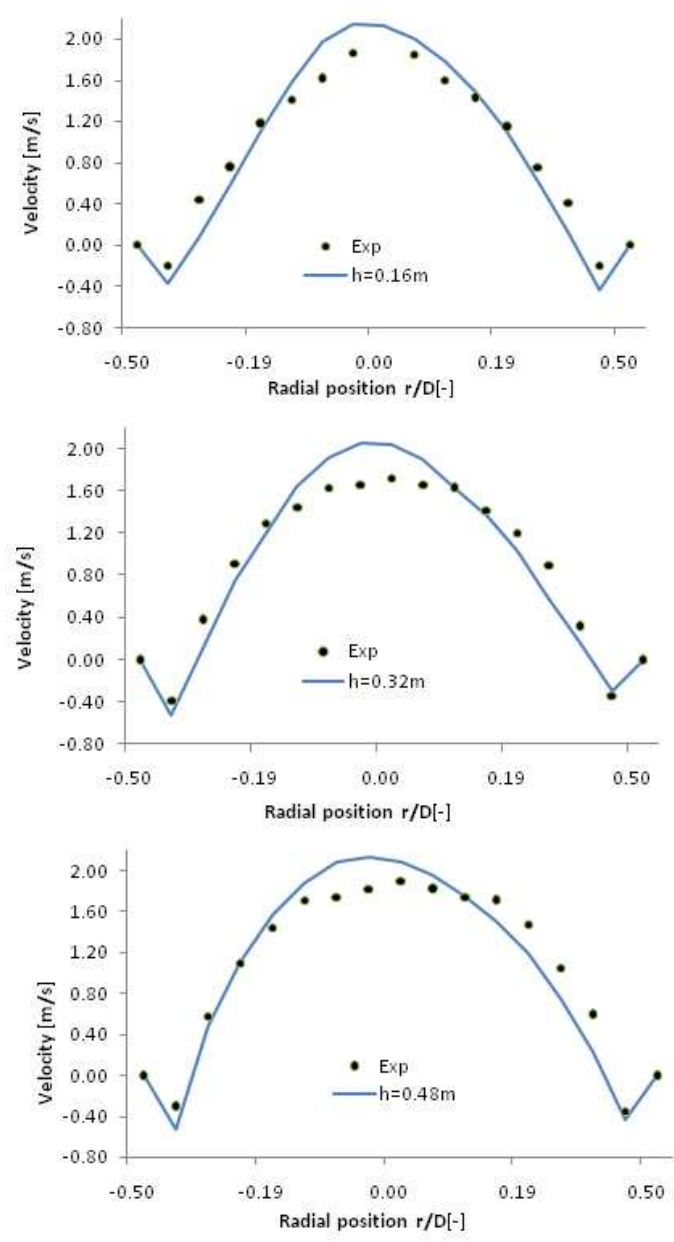

Figure 6: Experimental and simulated particle velocities at heights $0.16 \mathrm{~m}, 0.32 \mathrm{~m}$ and $0.48 \mathrm{~m}$ for $\mathrm{d}_{p}=60 \mu \mathrm{m}$ and $\mathrm{V}=$ $1.42 \mathrm{~ms}^{-1}$

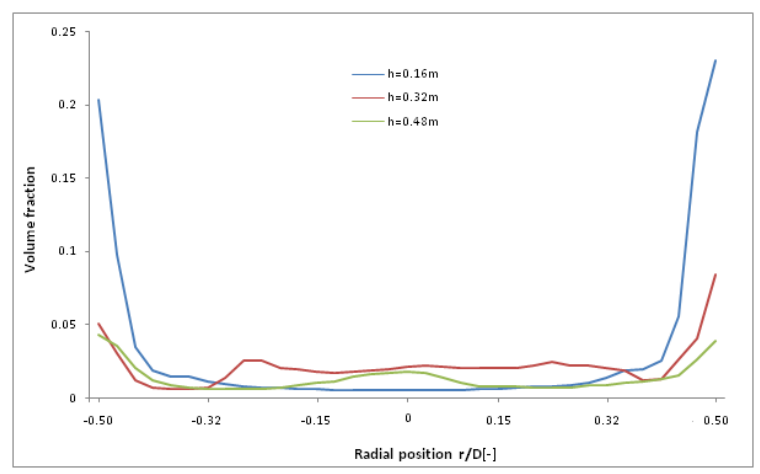

Figure 7: Radial profiles of particle volume fraction at heights of $0.16 \mathrm{~m}, 0.32 \mathrm{~m}$ and $0.48 \mathrm{~m}$ for $\mathrm{d}_{p}=60 \mu \mathrm{m}$ and $\mathrm{V}$ $=1.42 \mathrm{~ms}^{-1}$ 


\begin{tabular}{|l|l|l|l|}
\hline Radial Velocity (m/s) & Volume Fraction & Radial Velocity (m/s) & Volume Fraction \\
\hline 2.07 \\
1.93 \\
1.78 \\
1.64 \\
1.49 \\
1.35 \\
1.20 \\
1.06 \\
0.91 \\
0.77
\end{tabular}

Figure 8: 3D comparisons of the Gidaspow and EMMS drag models at the centre of the lower 0.4m riser. Contour plots of the radial velocities and volume fractions for each drag model with $\mathrm{d}_{p}=60 \mu \mathrm{m}$ and $\mathrm{V}=1.42 \mathrm{~ms}^{-1}$

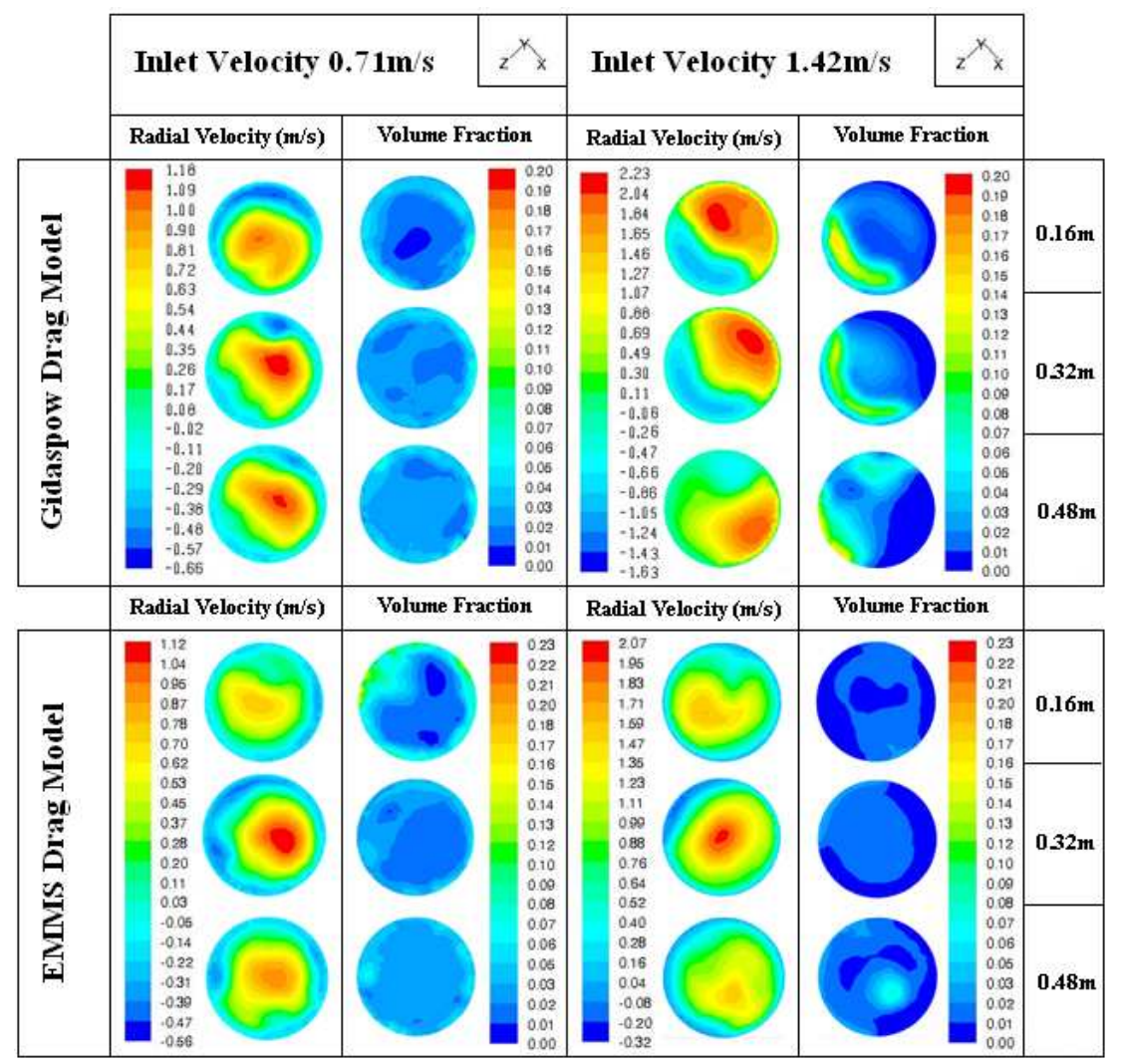

Figure 9: Comparing the 3D radial velocities and volume fraction of particles of both drag model across the heights $0.16 \mathrm{~m}$, $0.32 \mathrm{~m}$ and $0.48 \mathrm{~m}$ for $\mathrm{d}_{p}=60 \mu \mathrm{m}$ and velocities $\mathrm{V}=0.71 \mathrm{~ms}^{-1}$ and $\mathrm{V}=1.42 \mathrm{~ms}^{-1}$ 
within the dilute region which are carried with the flow. The Gidaspow model does not take into account the dense region at the base of the riser however a large cluster of particles is seen to travel along the side of the wall. This clustering effect has a significant implication on the flow distribution which causes an asymmetric flow structure. This would indicate the results from the $2 \mathrm{D}$ models are affected by the drag model's inablility to correctly take into account clustering effects. Whereas the flow structure of the EMMS model has the expected core annular distribution as you would expect. The EMMS flow structure appears to show slight asymetry which would be a result of the transitional effects.

The magnitude of the radial velocities in Fig. 8 agrees very well with the $2 \mathrm{D}$ experimental results in Fig. 6. The Gidaspow model continues to show an overpredicted core velocity due to the presence of a large cluster within the riser tube which reduce the area the air-particle dilute flow can travel through. In comparison, the EMMS drag model shows a better value for the core velocity. The radial velocities for the downflow of particles at the wall agree very well in both cases with the experimental results as the wall effects included a slight roughness.

The radial velocities in Fig. 9 were taken across three slices at heights $0.16 \mathrm{~m}, 0.32 \mathrm{~m}$ and $0.48 \mathrm{~m}$ for the velocities $0.71 \mathrm{~ms}^{-1}$ and $1.42 \mathrm{~ms}^{-1}$. The results show the presence of asymmetry with the faster velocities particularly for the Gidaspow drag model. This confirms that transitional effects would influence the flow structure and particle distribution. The asymmetric velocities are more apparent in the $3 \mathrm{D}$ results compared to the $2 \mathrm{D}$ results in Fig 6 and the previous paper [1]. The position of the secondary air inlet shows a slight influence on the flow for velocity $0.71 \mathrm{~ms}^{-1}$ at $0.16 \mathrm{~m}$ for the Gidaspow and inlet velocities of $0.71 \mathrm{~ms}^{-1}$ $1.42 \mathrm{~ms}^{-1}$ for the EMMS model. The flow starts to centralise with height, as expected.

The magnitudes of the radial velocities of the fastest particles coincide with those observed in Fig 6 and experiments with the highest velocities around $2.0 \mathrm{~ms}^{-1}$. The overestimation of the Gidaspow model is more apparent and confirms the collection of particles at the walls reduce the core diameter which would increase flow velocity. The downflow radial velocity of particles against the wall shows the majority of particles are falling at the wall down to $-0.532 \mathrm{~ms}^{-1}$; whilst the downflow radial velocity of particles for the EMMS model shows a lower velocity of around $-0.32 \mathrm{~ms}^{-1}$ which agrees with the experiemental results.

The volume fraction distributions in Fig. 9 for all cases show the accumulation of particles towards the walls. The lower velocities show an even distribution of particles around the walls of the riser and a lower distribution within the central region where the velocity is fastest. At lower heights the volume fraction of particles against the walls are slightly increased due to the continual collection of descending particles. This argees well with the 2D results seen in Fig. 7. The Gidaspow drag model at the $1.42 \mathrm{~ms}^{-1}$ shows a larger cluster of particles situated towards a single area of the wall where the velocity was lower. This does not agree with the 2D results observed in Fig. 6 and Fig. 7. The EMMS shows a more dilute distribution in particularly for faster velocity due to the majority of particle clusters being present at the base of the riser.

\section{Conclusion}

Two-dimensional and three-dimensional simulations were carried out on a circulating fluidised bed taken from literature[1]. The transition from a bubbling bed regime to a fast fluidising regime was considered for a variety of inlet velocities using the riser of the CFB. Results were as expected with the transition from bubbling to fast fluidising regimes occuring after the inlet velocity exceeded the terminal velocity. Within the bubbling regime, bubble size increased with height and also as the inlet velocity and particle diameter increased. The lower the velocity, the larger the collection of particles settling back to the base of the riser. The fast fluidising regime showed the segregation of particles towards the wall and the fast flowing core. The complete CFB geometry was simulated for a fast fluidising regime using two drag models, the Gidaspow and the energy minimisation multiscale (EMMS) model. The results agreed fairly well with the experimental results taken from literature[1] in both cases however the EMMS model correctly predicted the particle clusters which the Gidaspow model was unable to take into account. Whilst the Gidaspow results were slightly over estimated within the core the EMMS model showed an improvement. Improvements were made on the radial velocity of the particle downflow at the walls as the shear on the wall was slightly increased compared to previous results which imposed a free slip condition. Further 
exploration was extended to determine the volume fraction distribution of particles at three heights within the riser. $2 \mathrm{D}$ and $3 \mathrm{D}$ results agreed that higher volume fractions of particles are found at the walls, as seen experimentally, with an increase in volume fraction lower down the riser. The increase of airflow to a turbulent state along with the presence of the secondary inlet affects the flow of particles within the bed to produce an irregular flow lower down the riser becoming more regular with height. This is observed more clearly in the three-dimensional models.

\section{References}

[1] Samuelsberg, A., and Hjertager, B.H., 1995, "An experimental and numerical study of flow patterns in a circulating fluidized bed reactor." Int. J. Multiphase Flow. 22: 575-591

[2] Berruti, F., Chaouki, J., Godfroy, L., Pugsley, T.S., and Patience, G., 1995, "Hydrodynamics of circulating fluidized bed risers: a review." Canadian Journal of Chemical Engineering, 73(5): 579-602

[3] Ge, W., and Li, J., 1997, "Pseudo-particle approach to hydrodynamics of gas/solid two-phase flow." in Proceedings of the 5th International Conference on Circulating Fluidized Beds (eds. Li, J., Kwauk, M.), Beijing: Science Press.pp. 260265 .

[4] Ge, W., and Li, J., 2001, "Macro-scale pseudo-particle modeling for particle-fluid systems." Chinese Science Bulletin 46 18, pp. 15031507.

[5] Ge, W., and Li, J., 2003, "Macroscale Phenomena Reproduced in Microscopic Systems-Pseudo-Particle Modeling of Fluidization." Chemical Engineering Sciences. 58: 15651585

[6] Sundaresan, S., 2000, "Perspective:Modeling the hydrodynamics of multiphase flow reactors: current status and challenges." AIChE 46: 1102-1105

[7] Gidaspow, D., 1994, Multiphase flow and fluidization, continuum and kinetic theory descriptions. Academic Press, Inc. pp. 239-355

[8] Gidaspow, D., Bezburuah, R., Ding, J. 1992, "Hydrodynamics of Circulating Fluidized Beds: Kinetic Theory Approach." Presentation at 7th Fluidization Conference

[9] Syamlal, M., O'Brien, T.J. 1987, "Derivation of a drag coefficient from velocityvoidage correlation." U.S. Dept. of Energy, Office of Fossil Energy, National Energy Technology Laboratory, Morgantown, West Virginia April

[10] O'Brien, T.J., Syamlal, M. 1993, "Particle Cluster effects in the Numerical Simulation of a Circulating Fluidized Bed." In Preprint Volume for CFB-IV; Avidan, A.A.; Ed.; AIChE: New York. pp.430-435

[11] Gunn, D.J., Malik, A.A. 1967, "The Structure of Fluidized Beds in Particulate Fluidization." Proceedings of the International Symposiumon Fluidization; Dringkenbrug, A.A., Ed.; Netherlands University Press: Eindhoven, The Netherlands. pp. 52-65

[12] Yang, N., Wang, W., Ge, W., Wang, L. 2004, "Li J. Simulation of Heterogeneous Structure in a Circulating FluidizedBed Riser by Combining the Two-Fluid Model with the EMMS Approach." Industrial and Engineering Chemistry Research. 43:5548-5561
[13] Li, J.H., Chen, A., Yan, Z., Xu, G., Zhang, X. 1993, "Particle-Fluid Contacting in Circulating Fluidized Beds." In Preprint Volume for CFB-IV; Avidan, A. A., Ed.; AIChE: New York pp 49-54

[14] Li, J.H., Cheng, C., Zhang, Z., Yuan, J., Nemet, A., Fett, F.N. 1999, "The EMMS model-its application, development and updated concepts." Chemical Engineering Science 54: 5409-5425

[15] Jiradilok, V., Gidaspow, D., Breault, R.W. 2007, "Computation of gas and solid dispersion coefficients in turbulent risers and bubbling beds." Chemical Engineering Science 62: $3397-3409$

[16] Li, J.H., Wen, L., Ge, W., Cui, H., Ren, J. 1998, "Dissipative Structure in Concurrent-up Gas-Solid Flow." Chemical Engineering Science 53: 3367-3379

[17] Benyahia, S., Arastoopour, H., Knowlton, T.M., and Massah, H., 2000, "Simulation of Particles and Gas Flow Behaviour in the Riser Section of a Circulating Fluidized Bed using the Kinetic Theory Approach for the Particulate Phase." Powder Technology. 112 24-33

[18] Almuttahar, A., and Taghipour, F., 2008, "Computational fluid dynamics of high density circulating fluidized bed riser: Study of modeling parameters." Powder Technology. 185: $11-23$

[19] Mathiesen, V., Solberg, T., and Hjertager, B.H., 1999, "A numerical study of three-dimensional multiphase flow pattern in a riser." Werther, J., Ed. 6th International Conference on Circulating Fluidized Beds, Würzburg, Germany, August 24-27, pp 249-254.

[20] Zhang, D.Z., and VanderHeyden, W. B, 2001, "Highresolution three dimensional numerical simulation of a circulating fluidized bed." Powder Technol. 116: 133-141.

[21] Kuipers, J.A.M., and van Swaaij, W.P.M., 1999, "Simulation of threedimensional (3D) riser flow using kinetic theory of granular flow." Werther, J., Ed. 6th International Conference on Circulating Fluidized Beds, Würzburg, Germany, August 24-27, pp 267-273

[22] Ibsen, C.H., Solberg, T., and Hjertager B.H., 2001, "Evaluation of a Three-Dimensional Numerical Model of a Scaled Circulating Fluidized Bed." Industrial and Engineering Chemistry Research. 40 (23): 5081-5086

[23] Wen, YC; Yu, YH. 1966, "Mechanics of Fluidization." Chem. Eng. Prog. Symp. Ser. 62: 100

[24] Ergun, S. 1952, "Fluid Flow through Packed Columns." Chem. Eng. Prog. 48(2): 89-94

[25] Vasquez, S.A, and Ivanov, V.A., 2000, "A Phase Coupled Method for Solving Multiphase Problems on Unstructured Meshes." In Proceedings of ASME FEDSM'O0: ASME 2000 Fluids Engineering Division Summer Meeting, Boston

[26] Chapman, S., Cowling, T.G., 1970, "The Mathematical Theory of Non-Uniform Gases." 3rd Edition, Cambridge, UK.: Cambridge University Press

[27] Schaeffer, D.G. 1987, "Instability in the Evolution Equations Describing Incompressible Granular Flow." J. Diff. Equ. 66: $19-50$

[28] Lun, C.K.K., Savage, S.B., Jeffrey, D.J., Chepurniy, N., 1984, "Kinetic Theories for Granular Flow: Inelastic Particles in Couette Flow and Slightly Inelastic Particles in a General Flow Field." J. Fluid Mech. 140: 223-256

[29] Johnson, P., and Jackson, R., 1987, "Frictional-collisional constitutive relations for granular materials, with application to plane shearing." Journal of Fluid Mechanics. 176: 67-93 